\title{
Disseminated Extramedullary Plasmacytoma with Unusual Cardiac and Pancreatic Involvement- Literature Review and Rare Case Report
}

\author{
S. Babu Peter ${ }^{1}$ V. Raghu Nandhan ${ }^{1} \quad$ R. Sandhiya ${ }^{1}$ \\ ${ }^{1}$ Department of Radio-Diagnosis, Barnard Institute of Radiology, \\ Madras Medical College, Chennai, Tamil Nadu, India \\ Indian | Radiol Imaging 2021;31:701-706.
}

\author{
Address for correspondence S. Babu Peter, MD, DNB, FICR, \\ Department of Radio-Diagnosis, Barnard Institute of Radiology, \\ Madras Medical College, Chennai 01, Tamil Nadu, India \\ (e-mail: drbabupeter@gmail.com).
}

\begin{abstract}
Keywords

- cardiac MRI

- extramedullary plasmacytoma

- multifocal

Disseminated extramedullary plasmacytoma (EMP) is an unusual entity that has nonspecific imaging features at MRI. Nevertheless, knowledge of its imaging features and unusual locations might help radiologists to suspect it in the appropriate clinical scenario. Some noncontiguous EMPs might mimic lymphoma and MRI would be useful as a problem-solving tool in EMP and would help in treatment planning. We report an unusual cardiac mass in cardiac MRI, a detailed work-up of which led to the diagnosis of multifocal EMP involving the heart, ribs, pancreas, retroperitoneum, and soft tissues of the thigh, rarely reported in the literature.
\end{abstract}

\section{Key Messages}

Extramedullary plasmacytoma most commonly involves the head and neck. Infrequent sites include the gastrointestinal tract, liver, spleen, pancreas, lungs, thyroid, breast, testis, and skin. Primary cardiac involvement occurs extremely rare, usually involves one or both atria. Multifocal EMP with multiple site involvement synchronously has been rarely reported and limited literature on incidence.

\section{Introduction}

We report an unusual cardiac mass in cardiac MRI, detailed work-up of which led to the diagnosis of multifocal extramedullary plasmacytomas (EMP) involving the heart, ribs, pancreas, retroperitoneum, and soft tissues of the thigh, rarely reported in the literature and cardiac involvement was found among 3\% of all reported EMPs. Very few cases of cardiac plasmacytoma have been reported from 1977 to 2020 (around 50).

\section{Case History}

A 76-year-old female patient came to our hospital with complaints of abdominal pain and distension for 2 months and breathlessness for 1 month. An emergency echocardiogram was done, and it showed a heterochronic mass lesion noted in the right atrial cavity attached to the interatrial septum with thickened interventricular septum (-Fig. 1). ejection fraction was $64 \%$ with normal left ventricular systolic function. She was referred for cardiac magnetic resonance imaging in our department to rule out atrial thrombus. Cardiac MRI was done in a 3 Tesla MRI (Siemens Healthineers, Skyra, Erlangen, Germany) as per institute protocol. Morphology was evaluated with haste axial, trufi coronal, and axial sequences with cine axial stack and standard cardiac views. It showed a well-defined hypointense soft tissue intensity lesion in right atrial cavity attached to the interatrial septum and extending along the atrioventricular groove (-Fig. 2). Similar intense soft tissue thickening was noted in the interventricular septum.
DOI https://doi.org/ 10.1055/s-0041-1735498. ISSN 0971-3026. (c) 2021. Indian Radiological Association. All rights reserved. This is an open access article published by Thieme under the terms of the Creative Commons Attribution-NonDerivative-NonCommercial-License, permitting copying and reproduction so long as the original work is given appropriate credit. Contents may not be used for commercial purposes, or adapted, remixed, transformed or built upon. (https://creativecommons.org/ licenses/by-nc-nd/4.0/)

Thieme Medical and Scientific Publishers Pvt. Ltd., A-12, 2nd Floor, Sector 2, Noida-201301 UP, India 

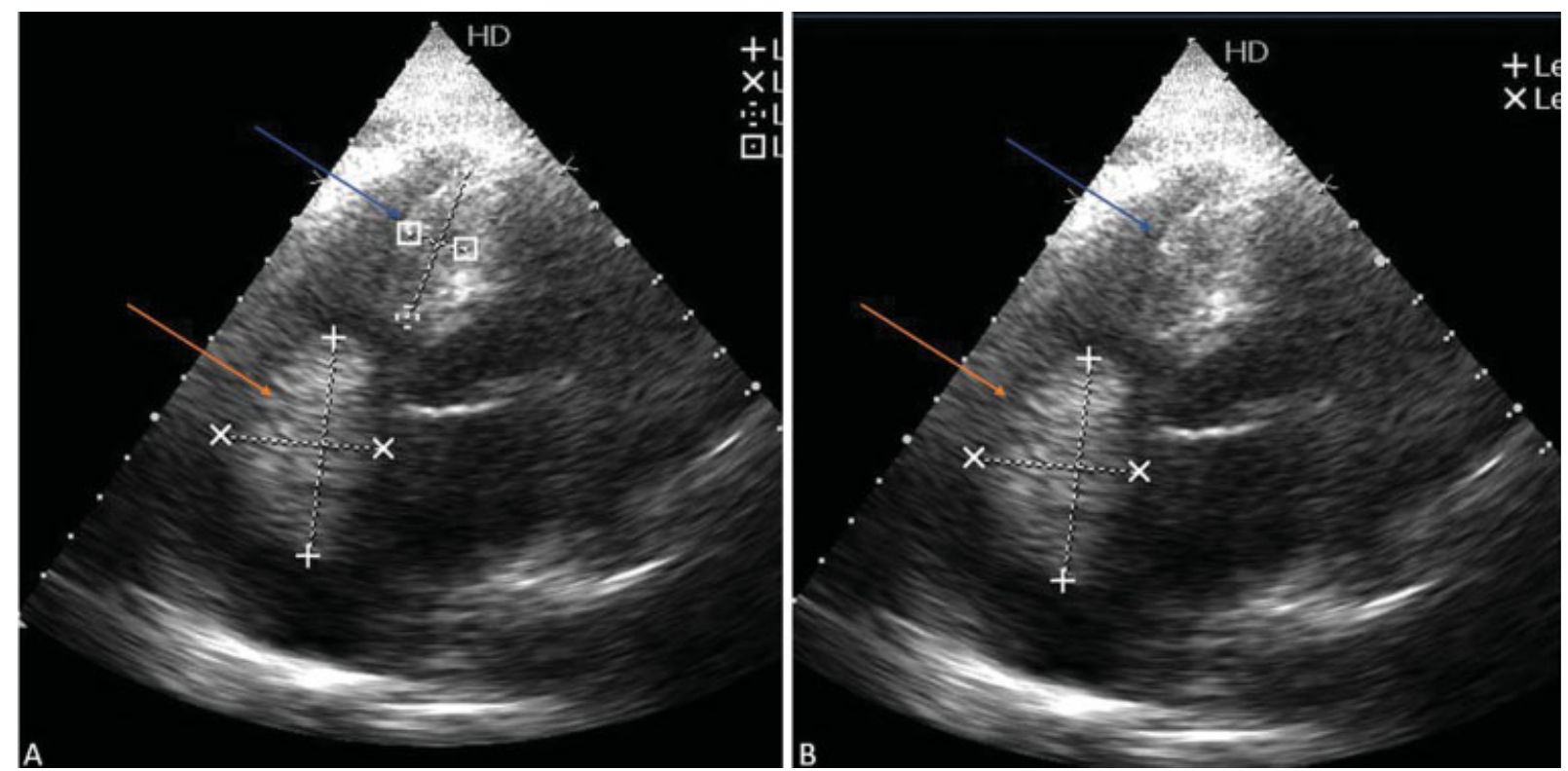

Fig. 1 Panels ( $\mathrm{A}$ and $\mathrm{B}$ ) show echocardiogram images of heteroechoic mass lesion in right atrium (orange arrow) attached to the interatrial septum. Note also the thickened interventricular septum (blue arrow).
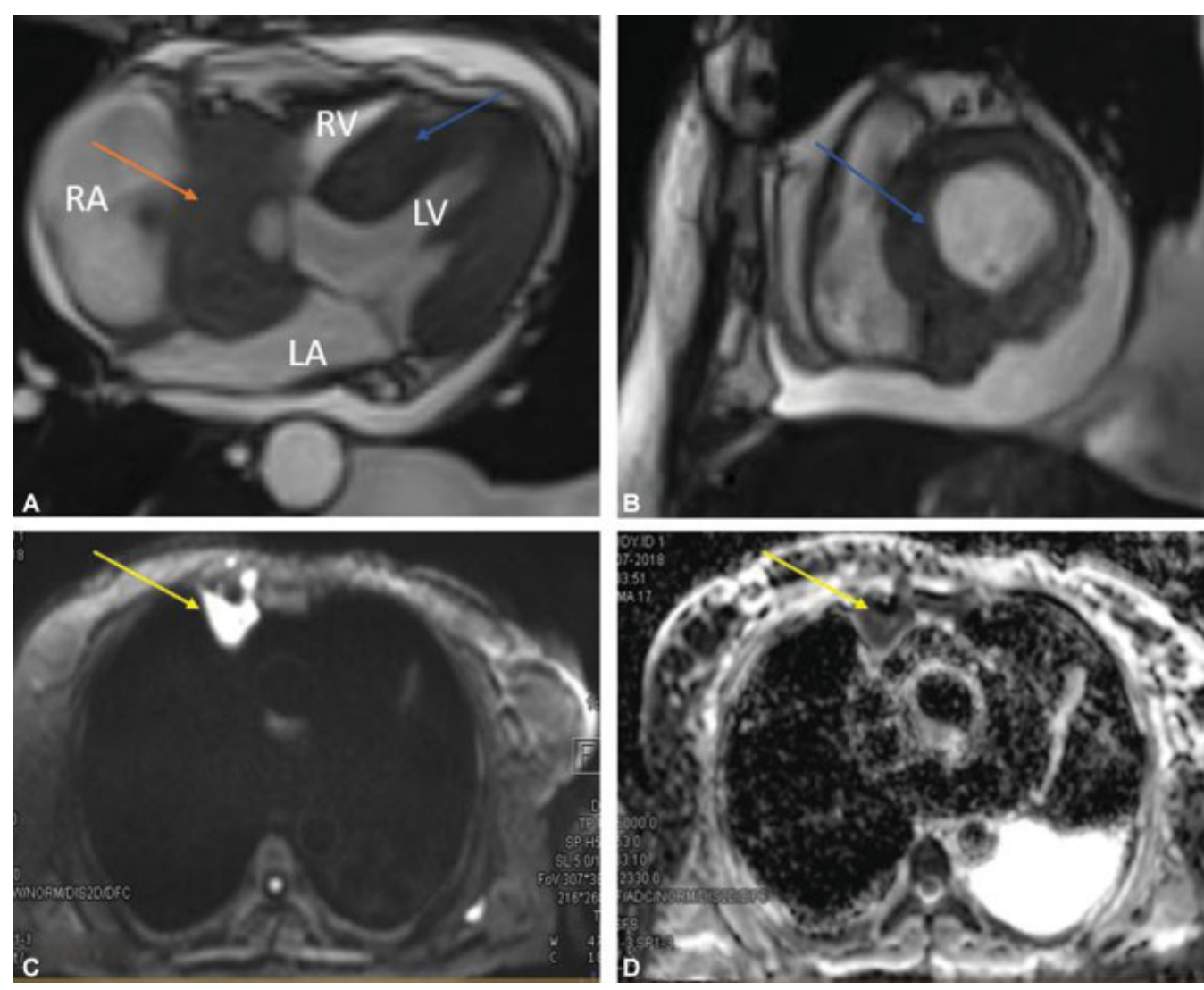

Fig. 2 (A) Cardiac MRI four-chamber view shows hypointense soft tissue intensity lesion noted in Interatrial septum (orange arrow) and atrioventricular groove. (B) Short axis view near base shows thickening of septal wall of left ventricle (blue arrow). (C and D) Diffusion and ADC sequences showing a lesion near right anterior chest wall showing diffusion restriction with low ADC values (yellow arrow). ADC, apparent diffusion coefficient.

Moderate bilateral pleural effusion was noted. Additionally, a soft tissue intensity lesion showing diffusion restriction and low apparent diffusion coefficient (ADC) values was noted in the right anterior chest wall. Axial images showed a hypointense lesion in the visualized abdominal sections and so the abdomen and pelvis were further evaluated. We found a large, lobular, relatively well-defined hypointense soft tissue intensity lesion in the left anterior pararenal 

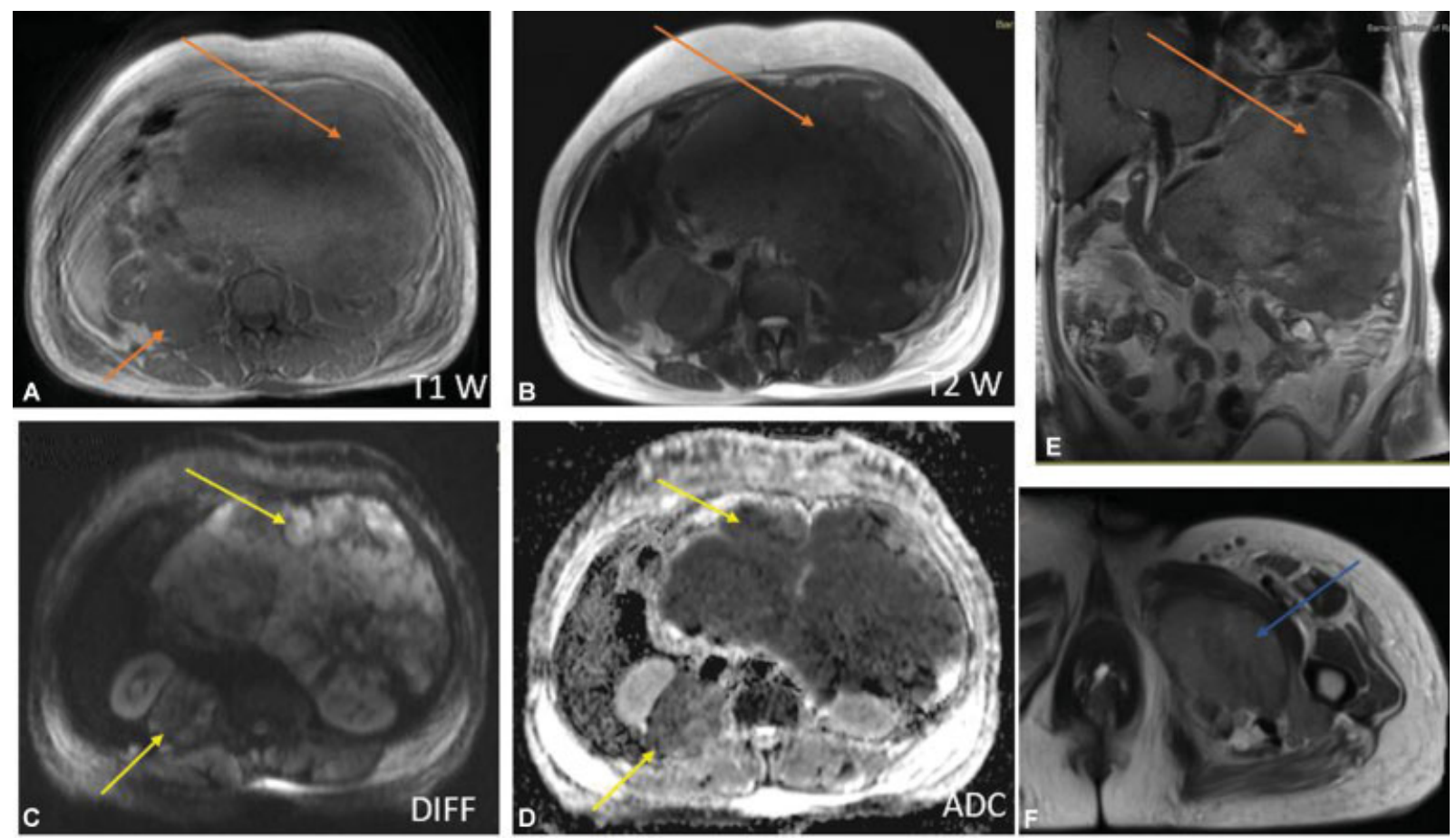

Fig. 3 (A and B) Axial T1W and T2W images hypointense lesion noted in left anterior pararenal space (orange arrow) infiltrating the pancreas and anterior wall of left kidney. Similar intense lesion noted in right paravertebral lesion. (C and D) Diffusion and ADC images show the lesion with restricted diffusion and low ADC values (yellow arrow). (E) Coronal haste image shows the large retroperitoneal lesion (orange arrow) with displacement of bowel loops. (F) Axial T2W haste image near the lower pelvis cut shows similar hypointense lesion in the medial thigh region (blue arrow).

space causing superior displacement of the spleen and medial displacement of bowel loops (-Fig. 3). The lesion also infiltrated the pancreas and anterior cortex of the left kidney. Similar signal intensity lesion was noted in right paravertebral region in posterior pararenal space. Both the lesions showed diffusion restriction and low ADC values. Surprisingly lower pelvic sections showed another similar signal intensity lesion in the medial left thigh in the intermuscular plane. CT chest was performed, and it showed cardiomegaly with moderate bilateral pleural effusion and CT abdomen with oral contrast showed a heterodense lesion with a similar extension (-Fig. 4). Visualized bones appeared normal. No evidence of enlarged mediastinal and abdominal lymph nodes was noted. Ultrasound screening of both abdomen and thigh showed large heteroechoic lesions with minimal peripheral vascularity (-Fig. 5) and biopsy was done from the retroperitoneal lesion. Histopathological findings were consistent with plasmacytoma and revealed numerous neoplastic plasma cells with nuclear pleomorphism. Immunohistochemistry staining showed CD 138 positivity and CK, CD 20, CD 3 negativity (-Fig. 6). Routine urine protein investigations were normal and serum IgG, IgA was increased mildly with no clinical significance $(1,400 \mathrm{mg} / \mathrm{dL}$ and $312 \mathrm{mg} / \mathrm{dL}$, respectively). Thus, a final diagnosis of multifocal EMP with a large retroperitoneal mass and involving the heart, anterior chest wall, right posterior pararenal space, and left thigh was made.

\section{Discussion}

Multiple myeloma (MM) is the most common primary bone malignant neoplasm affecting the age group between 40 and 80 years. $^{1}$ Advances in imaging modalities and treatment facilities increased the lifespan of patients. ${ }^{2}$ Extramedullary myeloma occurs in 7 to $18 \%$ of new cases as the primary manifestation and 6 to $20 \%$ of old MM cases. ${ }^{3}$ The mechanism of extramedullary occurrence might be a contiguous spread or hematogenous spread. ${ }^{4}$ The proliferation of plasma cells in bone or soft tissues, without bone marrow involvement or other systemic presentation of MM, is the feature of solitary bone plasmacytoma and extramedullary plasmacytoma. More than one extramedullary tumor of plasma cells or multiple sites of disease in bone is referred to as multiple EMP or multiple solitary plasmacytoma as seen in our case. ${ }^{5}$ EMPs can be disseminated at presentation as seen in our case.

Primary plasmacytoma, whether osseous or nonosseous, was distinguished from MM by the absence of hypercalcemia, renal insufficiency and anemia, normal skeletal survey, absence of bone marrow plasmacytosis, and serum or urinary paraprotein $<2 \mathrm{~g} / \mathrm{dL}^{6}$ Our case had normal calcium levels $(9.8 \mathrm{mg} / \mathrm{dL})$, normal bone marrow biopsy, and normal urinary protein levels. EMP most commonly involves the head and neck especially in the upper respiratory tract. ${ }^{7}$ Infrequent sites of involvement include the gastrointestinal tract, liver, spleen, pancreas, lungs, thyroid, breast, testis, or skin. EMP presenting as a soft tissue mass is, therefore, a rare 

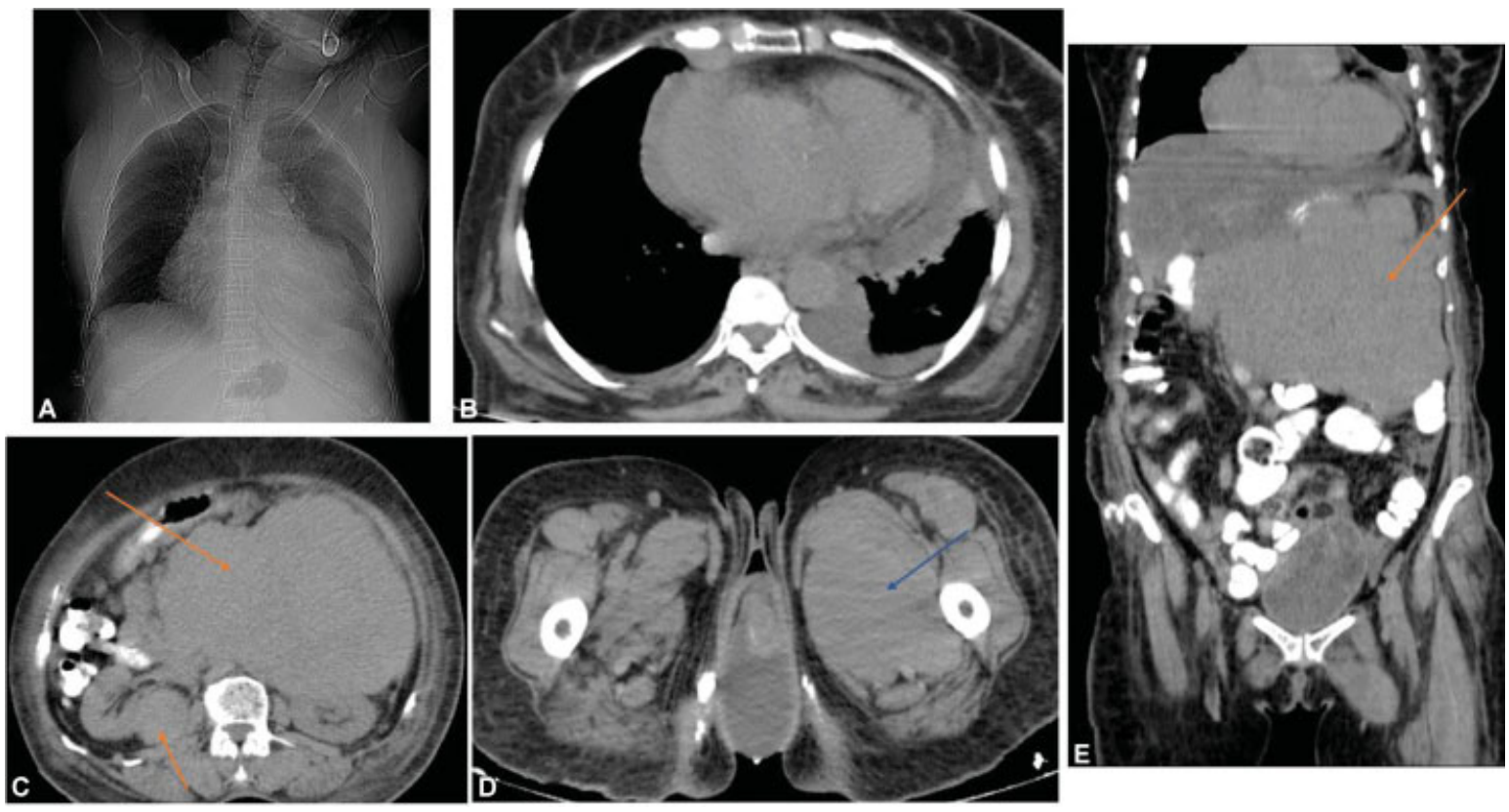

Fig. 4 (A and B) Plain CT scanogram and axial section show cardiomegaly and bilateral pleural effusion. (C) CT-abdomen axial section with oral contrast shows large hypodense lesion noted in left anterior pararenal space (orange arrow). Similar hypodense lesion noted in right paravertebral lesion. (D) Hypodense lesion in the medial thigh region (blue arrow). Panel (E) shows large lobular retroperitoneal lesion (orange arrow) with displacement of bowel loops.
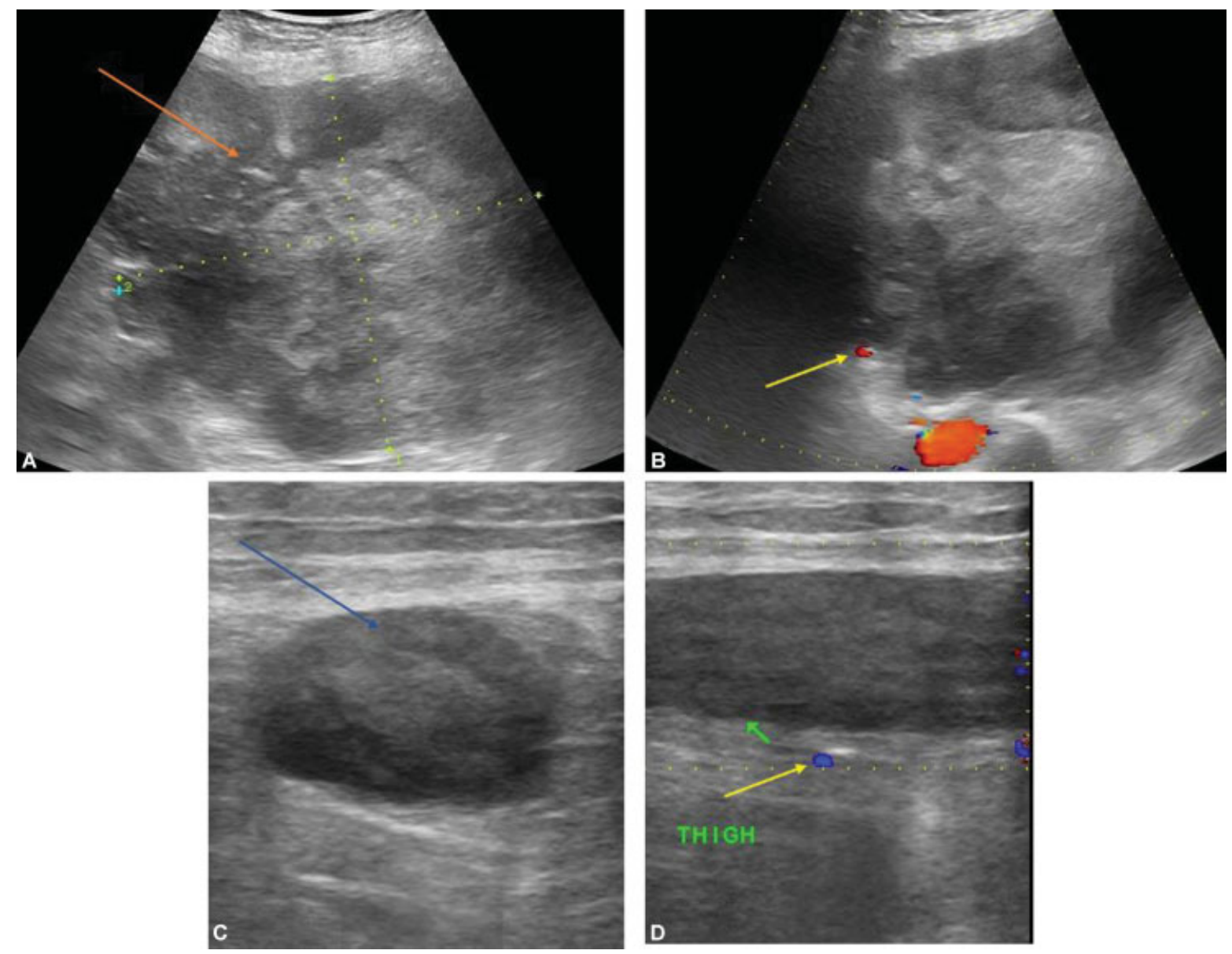

Fig. 5 (A and B) Ultrasound images of the abdomen show large heteroechoic lesion (orange arrow) with minimal peripheral vascularity (yellow arrow). (C and D) Ultrasound images of the thigh region show similar heteroechoic lesion (blue arrow) in intramuscular plane with minimal vascularity (yellow arrow). 

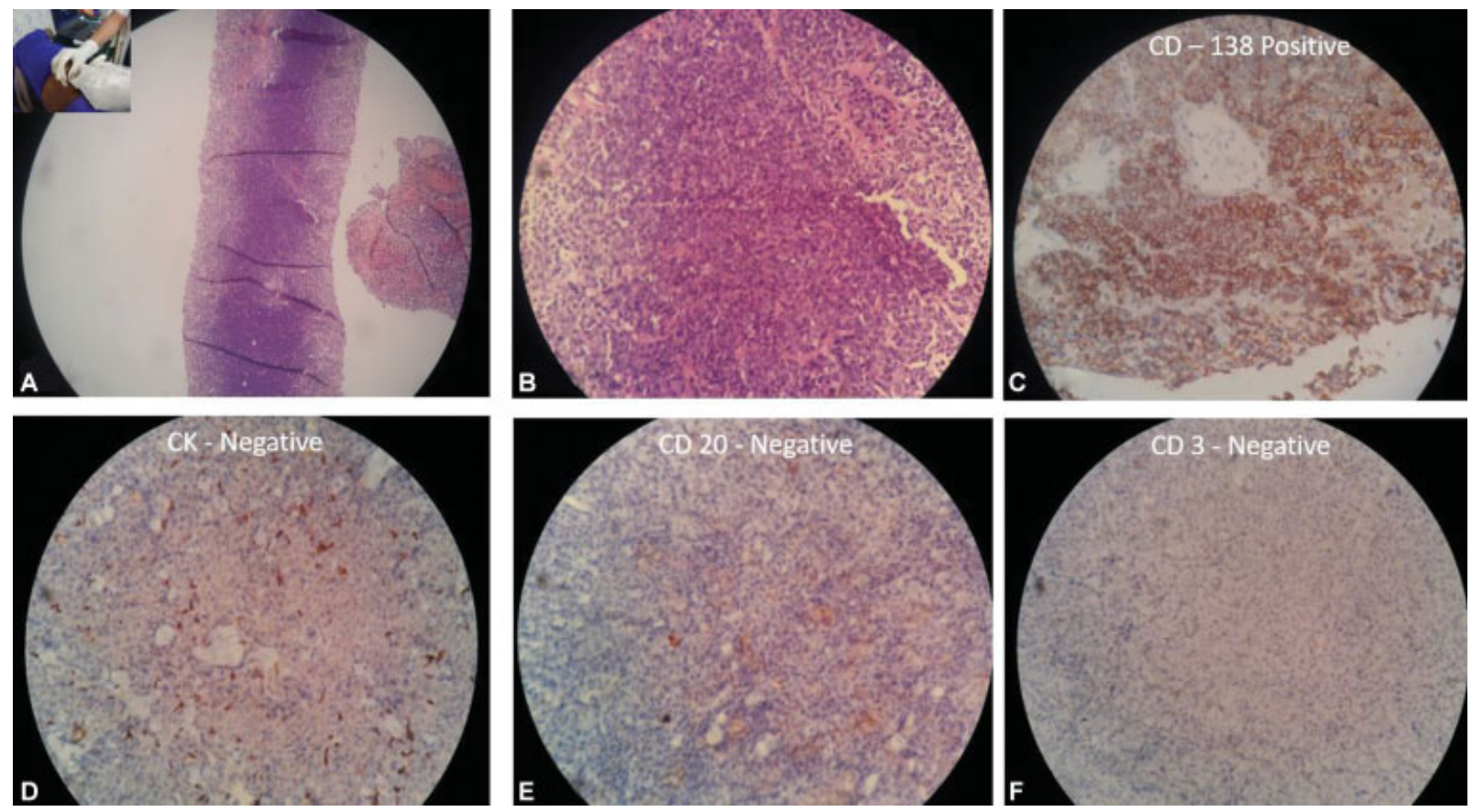

Fig. 6 (A) Low power field shows sheets of plasma cells. (B) High power field shows neoplastic plasma cells showing nuclear pleomorphism. (C-F) Immunohistochemistry staining shows CD-138 positivity and CK, CD 20, and CD 3 negativity.

event and not usually included in the differential diagnosis of other more common soft tissue masses, such as sarcomas, fibrous tumors, hemangiomas, neurofibromas, or lymphomas. But our case presented as multiple soft tissue masses in the retroperitoneum, chest wall, and thigh, necessitating histopathological and immunohistochemical assays to confirm the diagnosis.

Primary cardiac involvement occurs extremely rare and reported in $3 \%$ of cases and all cases showed involvement of one or both atria as large space-occupying lesions. ${ }^{8}$ Very few cases of cardiac plasmacytoma have been reported from 1977 to 2020 (around 50). ${ }^{9}$ Our case showed involvement of both atria as well as atrioventricular groove and interventricular septum. The right atrium is commonly involved in cases of disseminated plasmacytoma since it is postulated that circulating plasma cells can implant in the right atrium via venous route. ${ }^{10}$ This case also highlights the utility of cardiac magnetic resonance to localize, characterize, and evaluate cardiac masses and their physiologic effects besides providing excellent soft-tissue characterization. It also clearly shows the advantage of CMR over echocardiography in that MRI not only depicts the cardiac extent of mass but can be used to evaluate the abdomen in one go avoiding multiple visits by the patient.

Thus our case is peculiar in that the disease process involved retroperitoneum, thigh, and heart which were considered as unusual and uncommon sites of EMP. Multifocal EMP with the involvement of multiple sites at the same time has been rarely reported and there is limited literature on incidence and cross-sectional imaging. ${ }^{11}$ PubMed search revealed no cases of multifocal EMP with simultaneous involvement of the heart with retroperitoneum and thigh.
Our case is unique in not only this aspect but also the depiction of involvement by cardiac MRI.

\section{Conclusion}

Disseminated EMP is an unusual entity that has nonspecific imaging features at MRI. Nevertheless, knowledge of its imaging features and unusual locations might help radiologists to suspect it in the appropriate clinical scenario. Some noncontiguous EMP might mimic lymphoma and MRI would be useful as a problem-solving tool in EMP and helps in treatment planning. The possibility of EMP may be raised in the right atrial mass since it has a predilection for this site, although extremely rare when other associated findings exist. However, complete work-up is essential to rule out other lesions with multiplicities like lymphoma or metastases.

Financial Support and Sponsorship

None.

\section{Conflict of Interest}

None.

\section{References}

1 Hanrahan CJ, Christensen CR, Crim JR. Current concepts in the evaluation of multiple myeloma with MR imaging and FDG PET/CT. Radiographics 2010;30(01):127-142

2 Usmani SZ, Heuck C, Mitchell A, et al. Extramedullary disease portends poor prognosis in multiple myeloma and is over-represented in high-risk disease even in the era of novel agents. Haematologica 2012;97(11):1761-1767 
3 Varettoni M, Corso A, Pica G, Mangiacavalli S, Pascutto C, Lazzarino $\mathrm{M}$. Incidence, presenting features and outcome of extramedullary disease in multiple myeloma: a longitudinal study on 1003 consecutive patients. Ann Oncol 2010;21(02):325-330

4 Bladé J, Fernández de Larrea C, Rosiñol L, Cibeira MT, Jiménez R, Powles R. Soft-tissue plasmacytomas in multiple myeloma: incidence, mechanisms of extramedullary spread, and treatment approach. J Clin Oncol 2011;29(28):3805-3812

5 Aznab M, Khazaei M. Multifocal extramedullary and multiple solitary bone plasmacytoma: a case report and review of the literature. Int J Cancer Manag 2019;12:e91498

6 Ruiz Santiago F, Tello Moreno M, Martín Castro A, Guzmán Alvarez L, Navarrete González P. Soft tissue extramedullary plasmacytoma. Case Rep Med 2010;2010:307902

7 Serefhanoglu S, Sayinalp N, Haznedaroglu IC, et al. Extramedullary plasmacytomas of the thyroid and pericardium as initial presentation of multiple myeloma. Ann Hematol 2008;87(10): 853-854

8 Andrea R, Irene C, Armando F, De Vivo D, Simonetti G. Primary extramedullary plasmacytoma of the heart: a rare manifestation of plasmacellular tumor. Case Rep Radiol 2013; 2013:290849

9 Coakley M, Yeneneh B, Rosenthal A, Fonseca R, Mookadam F. Extramedullary cardiac multiple myeloma-a case report and contemporary review of the literature. Clin Lymphoma Myeloma Leuk 2016;16(05):246-252

10 Ooi GC, Chim JC-S, Au W-Y, Khong P-L. Radiologic manifestations of primary solitary extramedullary and multiple solitary plasmacytomas. AJR Am J Roentgenol 2006;186(03):821-827

11 Green J, Attwood C, Robbie H, Stefanidis K. Rare multifocal manifestation of solitary extramedullary plasmacytomas. BMJ Case Rep 2019;12(12):e232273 